\title{
Correlation Between Crystal Rotation and Redundant Shear Strain in Rolled Single Crystals: A Crystal Plasticity FE Analysis
}

\author{
Hui Wang ${ }^{1} \cdot$ Cheng Lu $^{1} \cdot$ Kiet Tieu $^{1} \cdot$ Yu Liu $^{1} \cdot$ Rui Wang $^{1} \cdot$ Jintao $\mathrm{Li}^{1}$ \\ Received: 30 August 2018 / Revised: 25 October 2018 / Published online: 6 December 2018 \\ (c) The Chinese Society for Metals (CSM) and Springer-Verlag GmbH Germany, part of Springer Nature 2018
}

\begin{abstract}
The correlation between crystal rotation and redundant shear strain in rolled single crystals was investigated by using the crystal plasticity finite element (CPFE) model in this paper. The deformation in aluminium single crystals of four representative orientations (rotated-Cube, Goss, Copper, and Brass) after rolling and plain strain compression was simulated, and the predictions have been validated by the experimental observations. In the rotated-Cube and Goss, the redundant shear strain and crystal rotation were in the same pattern, alternating along the thickness, while the relation between them was not obvious for the Copper and Brass due to their asymmetrical distributions of activated slip systems. The relations between slip system activation, crystal rotation, and shear strain were investigated based on the CPFE model, and the correlation between shear strain and crystal rotation has been built.
\end{abstract}

Keywords Crystal plasticity finite element $\cdot$ Single crystal $\cdot$ Crystal rotation $\cdot$ Shear strain $\cdot$ Orientation stability

\section{Introduction}

The deformation behaviours in single crystals after rolling and plain strain compression (PSC) have been extensively investigated (e.g. [1-7]). It has been found that the crystal rotation is very low in single crystals having stable orientations, including Copper $\{112\}\langle 111\rangle[1]$, Goss $\{011\}\langle 100\rangle$ $[2,3]$, and Brass $\{011\}\langle 211\rangle$ [4], while it is large in unstable orientations, such as Cube $\{001\}\langle 100\rangle[5]$ and rotated-Cube (roCube) $\{001\}\langle 110\rangle[6,7]$. An alternating pattern '+-+-' of crystal rotation along the thickness has been found in rolled Cube [8] and roCube [6], in which the four activated slip systems are symmetrically distributed about the rolling direction (RD)-transverse direction (TD) plane, and RD-normal direction (ND) plane. In contrast, the through-thickness crystal rotation does not exhibit any symmetry about the mid-thickness in single crystals that have asymmetrically distributed slip systems [1, 4]. As for shear strain, it is

Available online at http://link.springer.com/journal/40195

Hui Wang

hw737@uowmail.edu.au

1 School of Mechanical, Materials and Mechatronic Engineering, University of Wollongong, Wollongong, NSW 2522, Australia surprising that it is also in the ' +-+- ' pattern in Cube [9], which was experimentally measured by the embedded-pin method. However, the correlation between crystal rotation and shear strain in other orientations has not been observed, since measuring the redundant shear strain in single crystals is challenging [9].

As an alternative to experimental methods, texture modelling has become a powerful tool to study plastic deformation and texture evolution, and various crystal plasticity models have been developed. Homogenization at different levels has been adopted in the Taylor model, ALAMEL model, and self-consistent model [10]. Different from these models, no homogenization is assumed in the crystal plasticity finite element (CPFE) model, and thus, this model is in principle regarded as the best one in predicting plastic deformation and texture evolution $[11,12]$. The CPFE model incorporates the crystal plasticity constitutive model into the finite element (FE) framework, where the FE framework serves as the solver of boundary problems and the crystal plasticity constitutive law is taken into account at each integration point [13]. The equilibrium of stress and strain is achieved by the basic principles of mechanics at the same time for all material points [10]. The deformation in each element is determined by its current properties (e.g. crystal orientation) and external constraints (e.g. stress, strain). After an FE increment, the material properties are updated and used in 
the next increment. In this way, texture evolution and plastic deformation are fully coupled. According to the reports [14, 15], the main advantage of the CPFE model is its ability to solve crystal plasticity problems (e.g. slip system activation, texture evolution, and plastic deformation) under complicated internal and external boundary conditions.

In the authors' CPFE simulations of rolled single crystals, the crystal rotation has been found to be related to the redundant shear strain. The aim of this report is to study how they are correlated. The deformation in aluminium single crystals with four representative orientations (roCube, Goss, Copper, and Brass) has been predicted by the CPFE model. The slip system activation, shear strain, and crystal rotation have been investigated, and the relation between them was studied.

\section{Simulation Conditions and Texture Modelling}

Four simulation cases of rolling and PSC were carried out, as listed in Table 1, and the simulations were designed to match the experimental conditions. Cases $\mathrm{A}, \mathrm{B}$, and $\mathrm{C}$ were deformed by rolling (Fig. 1a) and 2D models (plain strain conditions) were used, since the main deformation was on the RD-ND plane. A 3D model was developed for the PSC in case D (Fig. 1b), since it is able to measure the shear strain on the RD-TD plane. The rolls in rolling (or dies in PSC) were considered as analytical rigid bodies, and the diameters are shown in Table 1 . The friction between the sheet and rolls was described by the Coulomb's friction law, and the friction coefficients were selected after testing various ones, since they generated the best-matched predicted textures. It was 0.25 for the unlubricated rolling in cases A and B, 0.11 for the lubricated rolling in case C, and 0.1 for the improved lubrication conditions in case $\mathrm{D}$. The coefficients of 0.25 and 0.11 match the suggested values for unlubricated and lubricated rolling [17], respectively. The element type was CPE4R in 2D models and C3D8R in the 3D model, which can provide an efficient and fast numerical formulation. Enhanced hourglass control was used to increase the resistance to the hourglassing problem and provide more accurate displacement solutions. The number of elements was determined by finding the element size at which the mechanical response became independent of the mesh resolution.

The starting materials were pure aluminium single crystals with initial orientations of roCube $\{001\}\langle 110\rangle[6]$, Goss $\{011\}\langle 100\rangle$ [3], Copper $\{112\}\langle 111\rangle[16]$, and Brass $\{011\}\langle 211\rangle[4] .\{001\}\langle 110\rangle$ is an unstable orientation in rolling, and its four potentially activated slip systems are shown in Fig. 2a. Figure $2 b$ shows the distribution of slip systems of Goss, a stable orientation. Copper is regarded as a semistable orientation in this study, since its crystal rotation after
Table 1 Simulation conditions of four simulation cases

\begin{tabular}{|c|c|c|c|c|}
\hline & Case A & Case B & Case $\mathrm{C}$ & Case D \\
\hline Initial orientation & roCube $(00 \overline{1})[110]$ & Goss (011)[100] & Copper (112)[111]] & Brass $(\overline{1} 01)[1 \overline{2} 1]$ \\
\hline Orientation stability & Unstable & Stable & Semi-stable & Stable \\
\hline Activated slip systems & Symmetrical & Symmetrical & Asymmetrical & Asymmetrical \\
\hline Deformation & Rolling & Rolling & Rolling & PSC \\
\hline Reduction & $50 \%$ & $30 \%$ & $50 \%$ & $43 \%$ \\
\hline Friction coefficient & 0.25 & 0.25 & 0.11 & 0.1 \\
\hline Diameter of rolls & $75 \mathrm{~mm}$ & $75 \mathrm{~mm}$ & $310 \mathrm{~mm}$ & $\infty$ \\
\hline Initial thickness & $3.0 \mathrm{~mm}$ & $2.8 \mathrm{~mm}$ & $4.0 \mathrm{~mm}$ & $7.0 \mathrm{~mm}$ \\
\hline Reference & [6] & {$[3]$} & [16] & {$[4]$} \\
\hline
\end{tabular}

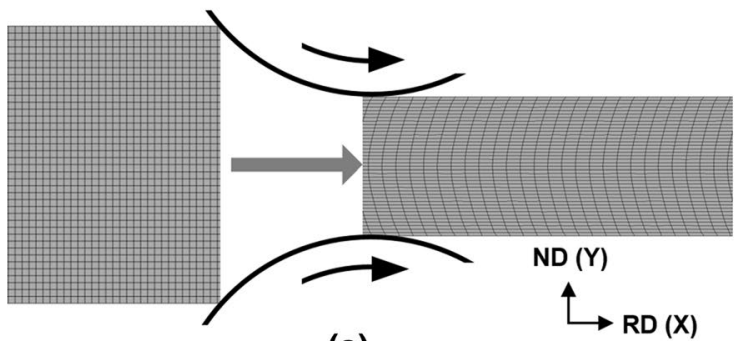

(a)

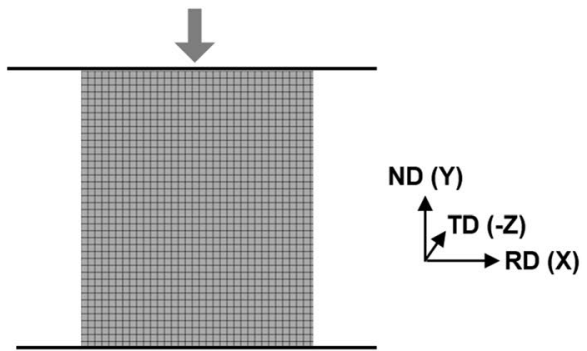

(b)

Fig. 1 a Rolling, b PSC model 


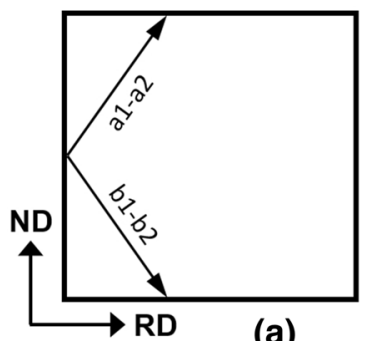

(a)

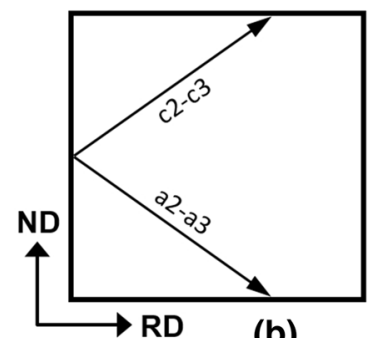

(b)
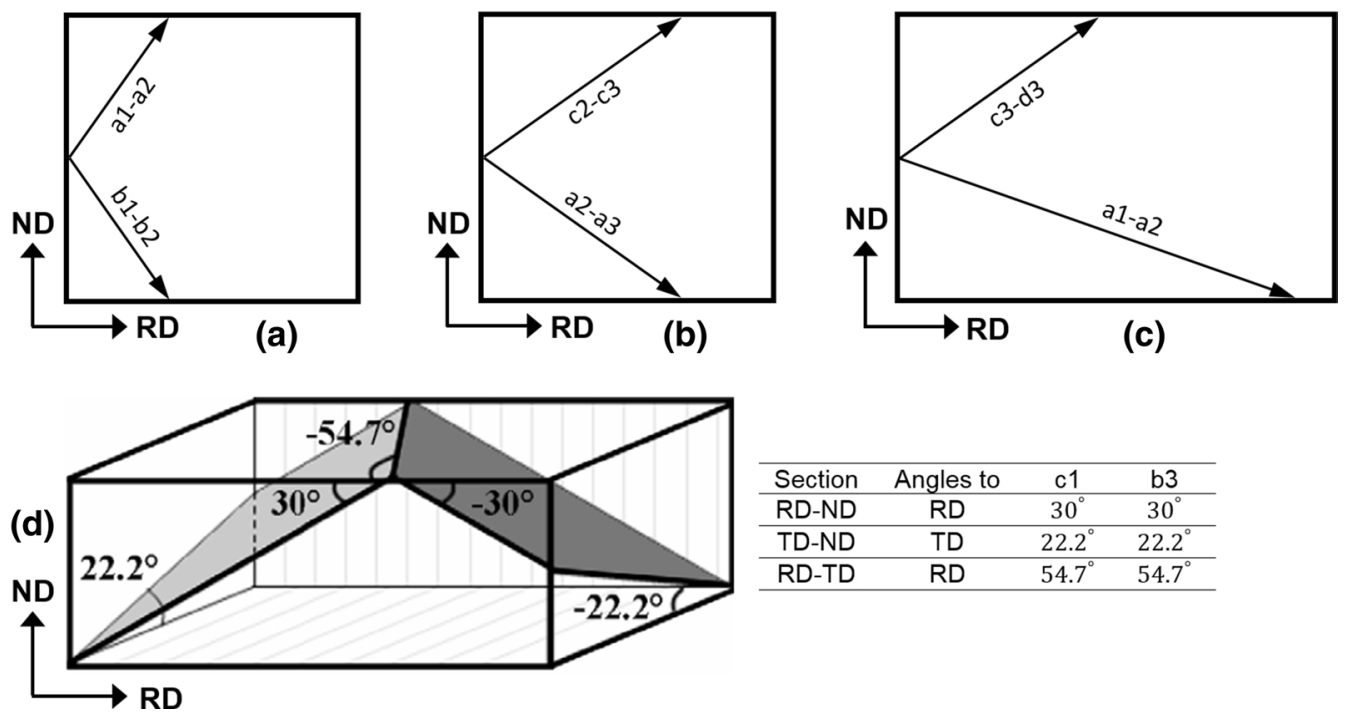

Fig. 2 Distribution of activated slip systems in a roCube, b Goss, c Copper, d Brass [18]

rolling is not small. Its four slip systems are symmetrical about the RD-ND plane, but not about the RD-TD plane (Fig. 2c). Brass is a stable orientation and its two slip systems are neither symmetrical about the RD-ND plane nor the TD-ND plane (Fig. 2d).

The kinematical scheme of the crystal plasticity model developed by Asaro [19, 20] was adopted in the present study, which has been widely used and recognized. A rate-dependent hardening model, Bassani-Wu hardening model [21, 22], was used, since it could accurately describe the material hardening [23]. In this hardening model, the shear strain rate $\dot{\gamma}^{(\alpha)}$ on slip system $\alpha$ is determined by its resolved shear stress $\tau^{(\alpha)}$, as described by Eq. (1), where $\dot{\gamma}_{0}^{(\alpha)}$ is the reference value of the shear strain rate, $\tau_{\mathrm{c}}^{(\alpha)}$ is the critical resolved shear stress, and $n$ is the rate-sensitive exponent of slip system $\alpha$. The values of $\dot{\gamma}_{0}^{(\alpha)}, n$ and $\tau_{\mathrm{c}}^{(\alpha)}$ are given in Table 2 .

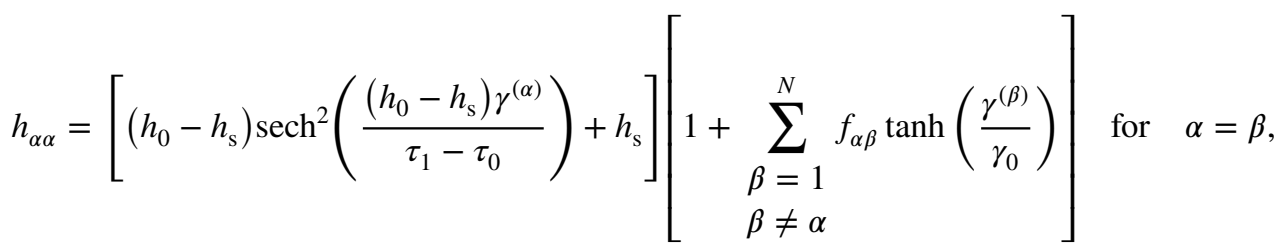

$\dot{\gamma}^{(\alpha)}=\dot{\gamma}_{0}^{(\alpha)} \operatorname{sgn}\left(\tau^{(\alpha)}\right)\left|\frac{\tau^{(\alpha)}}{\tau_{c}^{(\alpha)}}\right|^{n} \quad$ for $\quad \tau^{(\alpha)} \geq \tau_{c}^{(\alpha)}$

$\dot{\gamma}^{(\alpha)}=0$ for $\tau^{(\alpha)}<\tau_{c}^{(\alpha)}$,

$\operatorname{sgn}(x)= \begin{cases}1 & \text { for } \quad x \geq 0 \\ -1 & \text { for } \quad x<0\end{cases}$

The $\tau_{\mathrm{c}}^{(\alpha)}$ is the strength of activation of slip systems, and it increases with increasing strain according to

$\dot{\tau}_{c}^{(\alpha)}=\sum_{\beta=1}^{N} h_{\alpha \beta}\left|\dot{\gamma}^{(\beta)}\right|$

where $h_{\alpha \beta}$ is the hardening modulus. It is self-hardening, i.e. $h_{\alpha \alpha}$, when $\alpha$ is equal to $\beta$, while it is latent hardening $h_{\alpha \beta}$ when $\alpha$ is not equal to $\beta$. The $h_{\alpha \alpha}$ and $h_{\alpha \beta}$ are expressed by:
Table 2 Parameters used in the Bassani-Wu hardening model

\begin{tabular}{lllllll}
\hline$n$ & $\dot{\gamma}_{0}\left(\mathrm{~s}^{-1}\right)$ & $h_{0}(\mathrm{MPa})$ & $h_{\mathrm{s}}(\mathrm{MPa})$ & $\tau_{1}(\mathrm{MPa})$ & $\tau_{0}(\mathrm{MPa})$ & $q$ \\
\hline 300 & 0.0001 & 100 & 0.01 & 6.3 & 6 & 1 \\
\hline
\end{tabular}


$h_{\alpha \beta}=q h_{\alpha \alpha} \quad$ for $\quad \alpha \neq \beta$,

where $h_{0}$ is the hardening modulus after initial yield, $h_{\mathrm{s}}$ is the hardening modulus of easy slip, $\tau_{1}$ is the critical stress when plastic flow begins, $\tau_{0}$ is the initial critical resolved shear stress, $q$ is the ratio between latent hardening modulus and self-hardening modulus, and $f_{\alpha \beta}$ represents the interaction between two slip systems $\alpha$ and $\beta$. The value of $f_{\alpha \beta}$ is determined by the relative position of two slip systems. The parameter $f_{\alpha \beta}$ is chosen as: $a_{1}=a_{2}=a_{3}=1.75, a_{4}=2$, and $a_{5}=2.25$ [24]. Other material parameters used in the hardening models were evaluated by fitting the simulated stress-strain curve with the experimental results of an aluminium single crystal under PSC [25], and they are listed in Table 2. Three elastic moduli are $C_{11}=112,000 \mathrm{MPa}$, $C_{12}=66,000 \mathrm{MPa}$, and $C_{44}=28,000 \mathrm{MPa}$ [26].

The commercial FE code Abaqus/Standard version 6.9 was used to carry out the simulations, into which the crystal plasticity model was implemented by the user-defined material (UMAT) subroutine. The materials used in this study were aluminium single crystals of FCC structure, and the slip plane and slip direction were $\{111\}$ and $\langle 110\rangle$, respectively. The crystal plasticity theory, CPFE implementation, hardening model, and material parameters have been given in Ref. [12]. The combination of this CPFE model and the set of material parameters has accurately captured the texture evolution under different processes [12, 27, 28].

\section{Results}

\subsection{Case A: roCube}

Following the method used in the corresponding experimental research [6], the crystal rotation relative to the initial orientation was partitioned into TD-, RD-, and ND-rotations. The TD-rotation is strongly dominant over RD- and
ND-rotations, and accordingly, only TD-rotation is shown in Fig. 3. The predicted through-thickness TD-rotation in Fig. 3b matches well with the experimental measurement in Fig. 3a [6]. Though the reduction was only $50 \%$, the evolved TD-rotation is very high, with a maximum value of $28^{\circ}$, and thus, roCube is regarded as unstable in rolling [6, 9]. The direction of TD-rotation alternates along the thickness and divides the thickness into four matrix bands $(\mathrm{M})$ : M1-M2-M1-M2. The TD-rotation is in the positive (clockwise) direction in M1, while it is in the negative (anticlockwise) direction in M2. The denotation of matrix bands in the simulation is slightly different from that in Fig. 3a, where the experimentally observed region is noticeably smaller than the whole thickness [6]. It is surprising that the macroscopic redundant shear strain $\left(\gamma_{X Y}\right)$ distributes in the same pattern as TD-rotation, but they are in the opposite direction, since it is $-\gamma_{X Y}$ in Fig. 3b. The shear strain $\gamma_{X Y}$ is very low in this case considering dry rolling conditions.

Four slip systems shown in Fig. 2a were activated in the roCube, the same as those in the experiment [6], and they

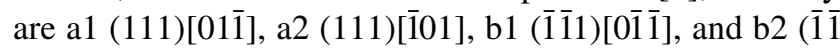
1)[101]. The shear strain on a1 $\left(\gamma_{\mathrm{a} 1}\right)$ is equal to $\gamma_{\mathrm{a} 2}$ due to their symmetries (Fig. 2a), and it is the same for b1 and b2. Therefore, only $\gamma_{\mathrm{a} 1}$ and $\gamma_{\mathrm{b} 1}$ are shown in Fig. $4 \mathrm{a}$, while Fig. $4 \mathrm{~b}$ represents the imbalance ratio between $\gamma_{\mathrm{a} 1}$ and $\gamma_{\mathrm{b} 1},\left(\gamma_{\mathrm{a} 1}-\gamma_{\mathrm{b} 1}\right.$ )$/ \max \left(\gamma_{\mathrm{a} 1}, \gamma_{\mathrm{b} 1}\right)$. The whole thickness in Fig. $4 \mathrm{a}, \mathrm{b}$ is also divided into four matrix bands, where $\gamma_{\mathrm{b} 1}$ is higher than $\gamma_{\mathrm{a} 1}$ in M1, while $\gamma_{\mathrm{b} 1}$ is lower in M2. The imbalance ratio is in the same direction as shear strain (Fig. 3b), and its value is very high, almost 1.0 at some points (near the centre) that possess large TD-rotation.

\subsection{Case B: Goss}

In this case, the partitioned TD-rotation agrees well with the experimental observation [3], as shown in Fig. 5. The
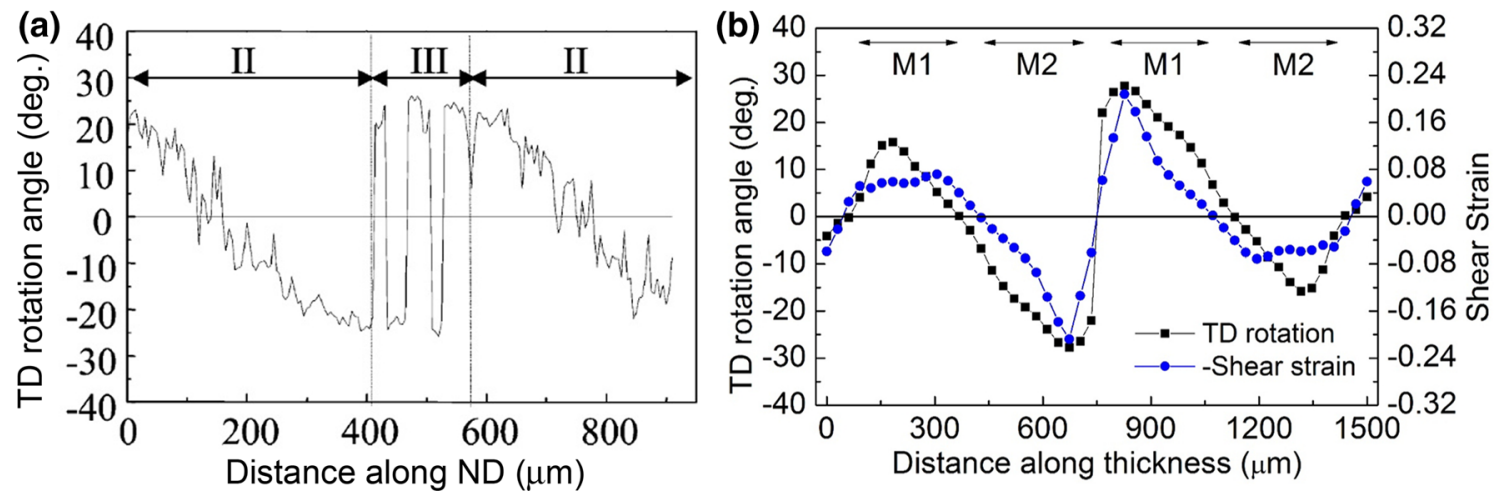

Fig. 3 Distrbiution of a TD-rotation in the experiment, $\mathbf{b}$ TD-rotation and $-\gamma_{X Y}$ in the simulation of roCube after a $50 \%$ reduction 

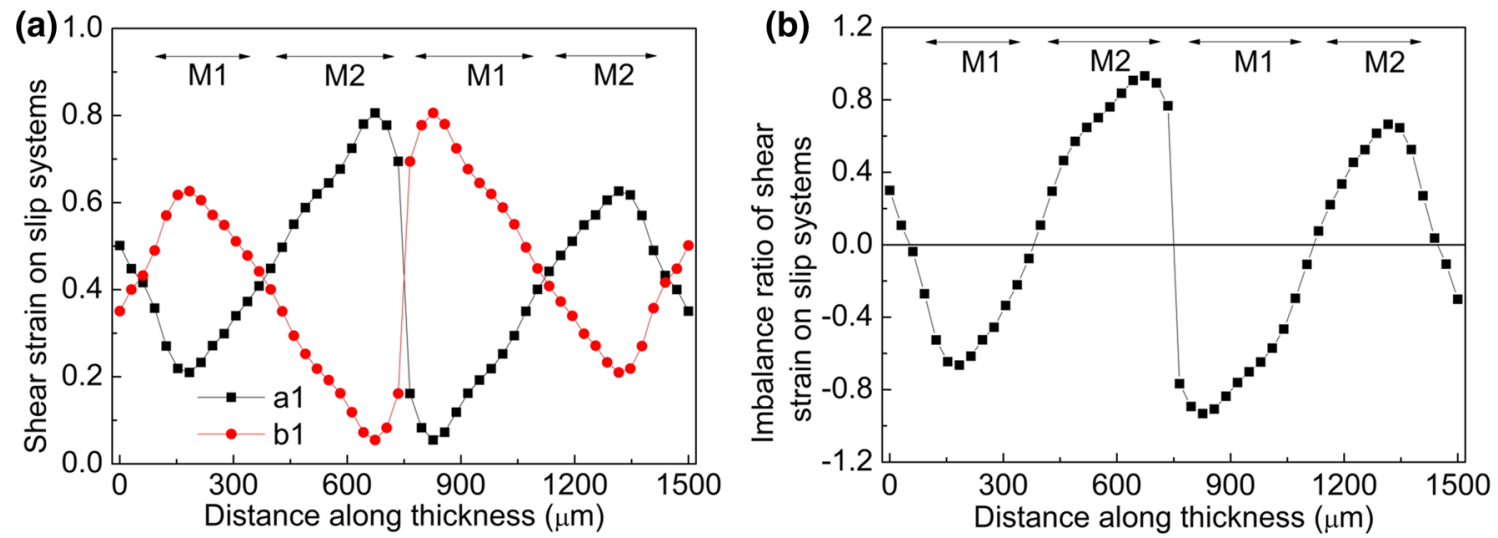

Fig. 4 Distribution of a shear strain on slip system a1 $\left(\gamma_{\mathrm{a} 1}\right)$ and $\mathrm{b} 1\left(\gamma_{\mathrm{b} 1}\right), \mathbf{b}$ imbalance ratio between $\gamma_{\mathrm{a} 1}$ and $\gamma_{\mathrm{b} 1},\left(\gamma_{\mathrm{a} 1}-\gamma_{\mathrm{b} 1}\right) / \mathrm{max}\left(\gamma_{\mathrm{a} 1}, \gamma_{\mathrm{b} 1}\right)$

(a)

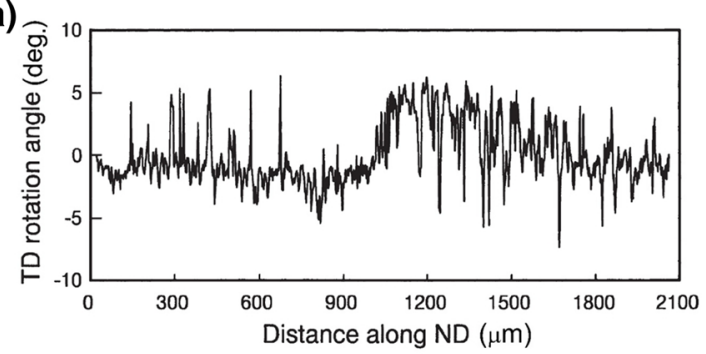

(b)

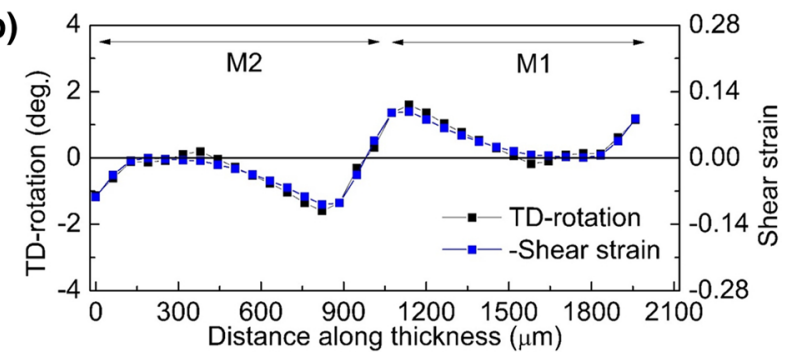

Fig. 5 Distribution of a TD-rotation in the experiment [3], b TD-rotation and $-\gamma_{X Y}$ in the simulation of Goss after a 30\% reduction maximum TD-rotation is only $1.6^{\circ}$, and thus, Goss is regarded as stable [2, 3]. Only two matrix bands developed in the Goss, and the TD-rotation is negative and positive in M2 and M1, respectively. The macroscopic shear strain, $-\gamma_{X Y}$, is in the same pattern as TD-rotation and its value is also very low (Fig. 5b).

The four activated slip systems in the Goss are a2 (111)[i 01], a3 (111)[11̄0], c2 (1̄11)[101], and c3 (1̄11)[1̄10] (Fig. 2b). The shear strain on $\mathrm{c} 2$ is equal to that on $\mathrm{c} 3$, and this is also true for $\mathrm{a} 2$ and a3. Only $\gamma_{\mathrm{a} 2}$ and $\gamma_{\mathrm{c} 2}$ are shown in Fig. 6a, and the imbalance ratio calculated according to $\left(\gamma_{\mathrm{c} 2}-\gamma_{\mathrm{a} 2}\right) / \mathrm{max}$ $\left(\gamma_{\mathrm{c} 2}, \gamma_{\mathrm{a} 2}\right)$ is shown in Fig. 6b. The activation of slip systems also divides the thickness into two matrix bands, where $\gamma_{c 2}$ is higher than $\gamma_{a 2}$ in M2, while $\gamma_{a 2}$ is dominant over $\gamma_{c 2}$ in M1. Accordingly, the imbalance ratio is positive in M2 (Fig. 6b), while negative in M1. The imbalance ratio distributes in a similar pattern to shear strain and TD-rotation in Fig. 5b, in the same direction as the former and opposite direction as the latter.
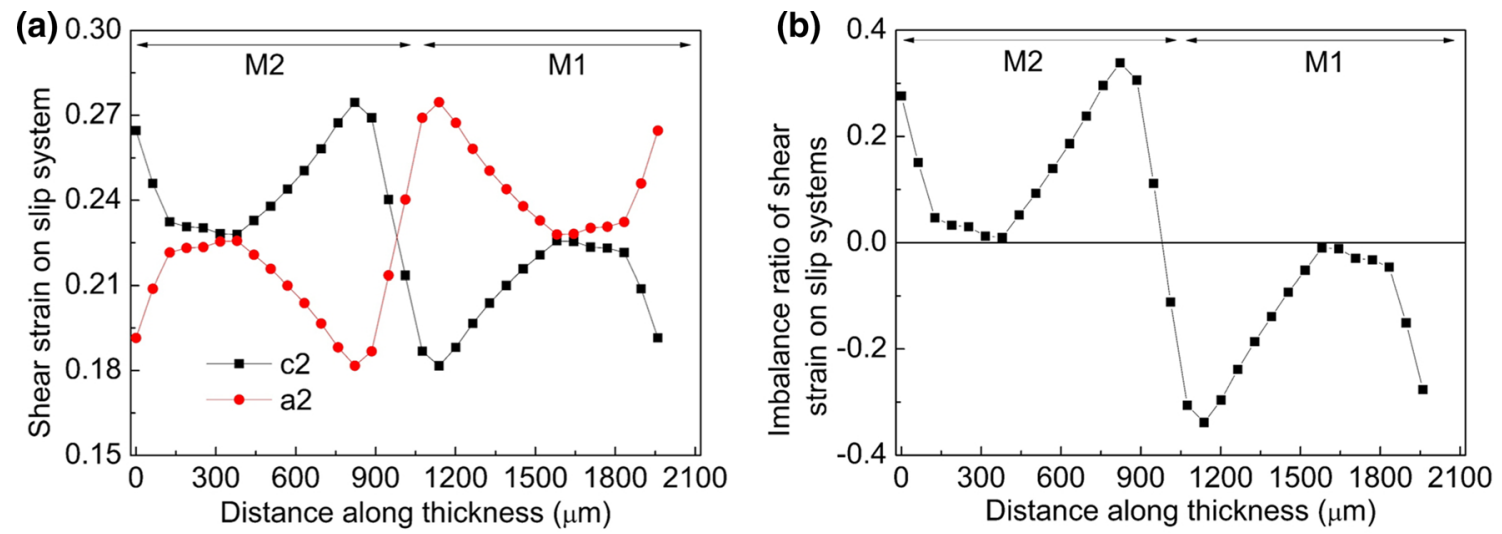

Fig. 6 Distribution of a shear strain on slip system c2 $\left(\gamma_{\mathrm{c} 2}\right)$ and a2 $\left(\gamma_{\mathrm{a} 2}\right), \mathbf{b}$ imbalance ratio between $\gamma_{\mathrm{c} 2}$ and $\gamma_{\mathrm{a} 2},\left(\gamma_{\mathrm{c} 2}-\gamma_{\mathrm{a} 2}\right) / \mathrm{max}\left(\gamma_{\mathrm{c} 2}, \gamma_{\mathrm{a} 2}\right)$ 

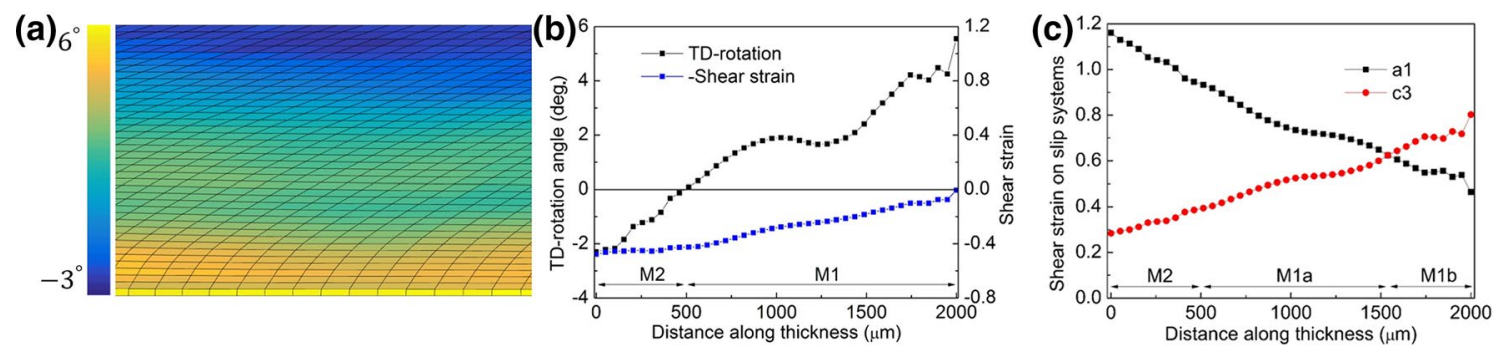

Fig. 7 Distribution of a TD-rotation and deformed FE mesh, b TD-rotation and $-\gamma_{X Y}, \mathbf{c}$ shear strain on slip system a1 $\left(\gamma_{\mathrm{a} 1}\right)$ and c3 $\left(\gamma_{\mathrm{c} 3}\right)$ along the thickness
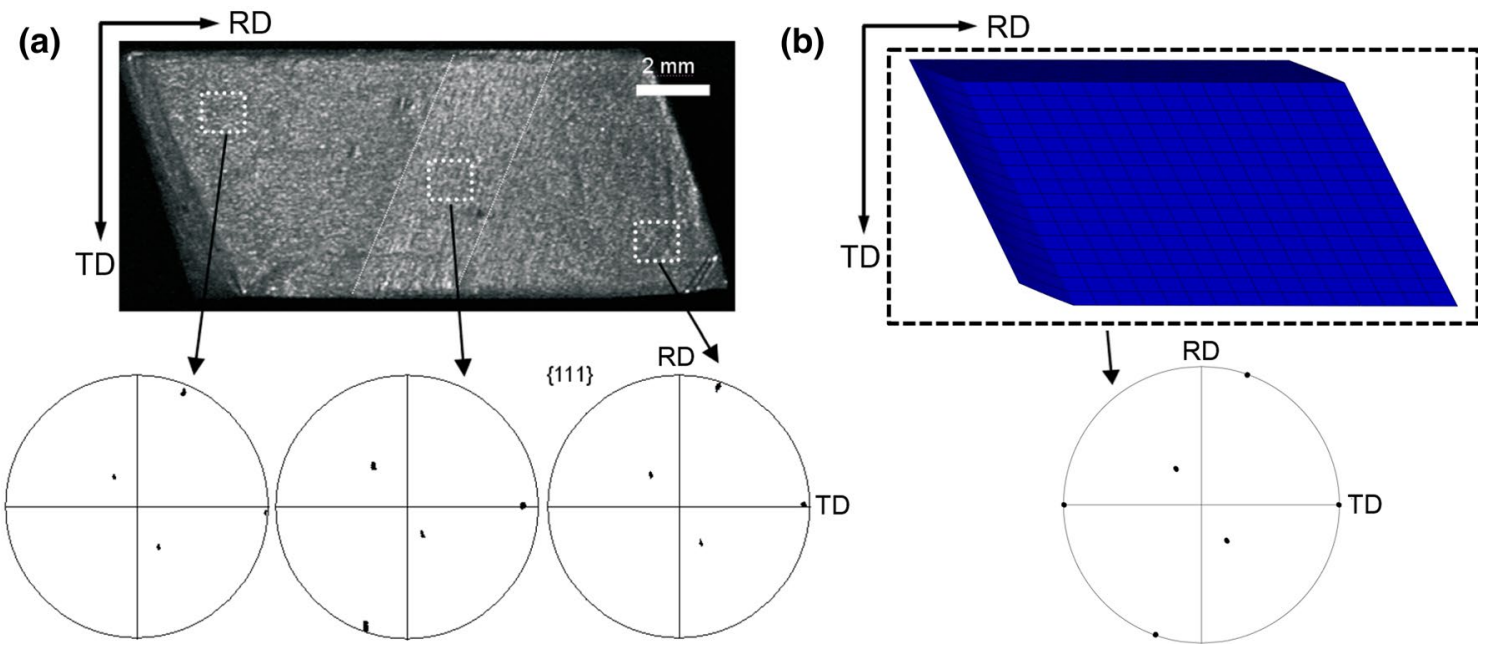

Fig. 8 Sample shape changes and $\{111\}$ pole figures in the a experiment [4], b simulation

\subsection{Case C: Copper}

The distribution of TD-rotation and shear strain (represented by the deformed FE mesh) shown in Fig. 7a is also plotted in Fig. $7 \mathrm{~b}$. The shear strain is positive along the whole thickness, but its value is almost zero at the lower surface. Like $\gamma_{X Y}$, the TD-rotation is also not symmetrical about the mid-thickness. The whole thickness is also divided into two matrix bands by the TD-rotation: M1 and M2. The four activated slip systems are a1 (111)[011̄], a2 (111)[ī01], c3 (ī11) [1̄ 1 10], and d3 (1 1 11)[110] (Fig. 2c). Slip system a1 and a2 are co-planar, and c3 and $\mathrm{d} 3$ are co-directional. The shear strain on 11 and a 2 are the same, and $\gamma_{\mathrm{c} 3}$ is equal to $\gamma_{\mathrm{d} 3}$. The activation of slip systems further partitions M1 into M1a and M1b (Fig. 7c). In M1a, $\gamma_{\mathrm{a} 1}$ is higher than $\gamma_{\mathrm{c} 3}$, while $\gamma_{\mathrm{c} 3}$ is relatively higher in M1b. This distribution of TD-rotation, shear strain, and slip system activation has been observed in other rolled aluminium single crystals of Copper orientation [16, 29].

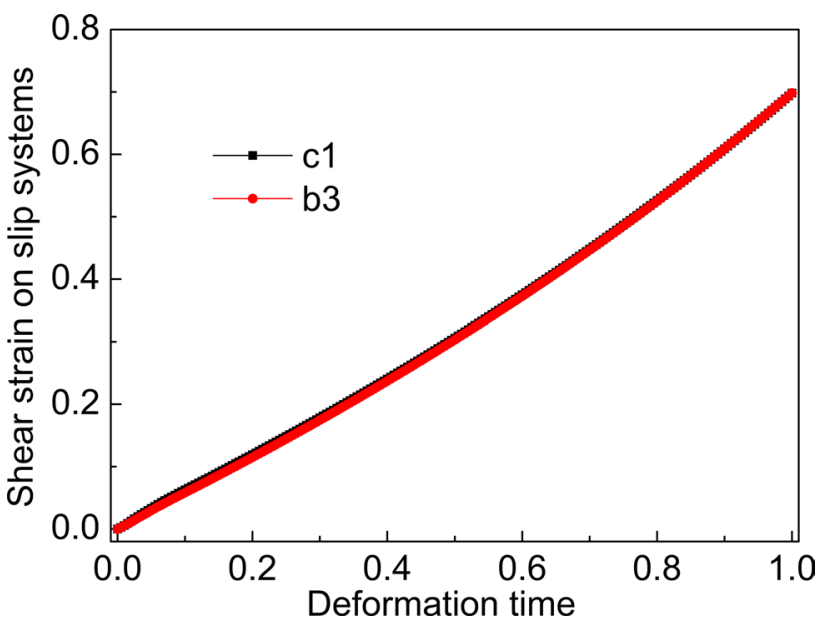

Fig. 9 Evolution of shear strain on slip system $\mathrm{c} 1\left(\gamma_{\mathrm{c} 1}\right)$ and $\mathrm{b} 3\left(\gamma_{b 3}\right)$ in one representative element 


\subsection{Case D: Brass}

As for the Brass orientation, a large change in the sample geometry on the RD-TD plane has been revealed in the experiment [4] and simulation (Fig. 8), but the deformation is almost uniform within the sample. The shear strain $\gamma_{X Z}$ is about 0.29 after a $43 \%$ reduction. The crystal rotation is very low in the experiment, as expressed by the $\{111\}$ pole figures from three regions (Fig. 8a). A similar texture was predicted in Fig. 8b, where the pole figure was constructed from all elements.

Only two slip systems, c1 (111)[011] and b3 (ī111)[1110], were activated in the Brass. Figure 9 shows the evolution of shear strain on these two slip systems as a function of deformation time in one representative element. It can be seen that $\gamma_{\mathrm{c} 1}$ and $\gamma_{b 3}$ increase gradually with the deformation time.

\section{Discussion}

The predictions match well with the experimental observations in cases $\mathrm{A}, \mathrm{B}$, and $\mathrm{D}$, which demonstrates that the developed CPFE model can predict accurate textures in rolling and PSC, and thus, the prediction in case $\mathrm{C}$ can be trusted though it has not been well validated. The large difference in deformation behaviours between the four orientations was caused by the difference in initial orientations [30, 31]. Four slip systems were activated in the roCube, Goss, and Copper, but only two in the Brass. In the roCube and Goss, TD-rotation and shear strain are both in an alternating pattern along the thickness, which suggests a potential relation between them. This potential relation in all four cases will be investigated as follows.

In the CPFE, the material spin $\boldsymbol{\Omega}$ can be represented by macroscopic shear strain [32], and it can be decomposed into plastic spin $\boldsymbol{\Omega}^{\mathrm{P}}$ and lattice spin (or crystal rotation) $\boldsymbol{\Omega}^{*}$, namely

$\boldsymbol{\Omega}=\boldsymbol{\Omega}^{\mathrm{P}}+\boldsymbol{\Omega}^{*}$.
The plastic spin $\boldsymbol{\Omega}^{\mathrm{P}}$ is caused by crystal slip, during which the crystal orientation keeps unchanged. The plastic spin is calculated by

$\boldsymbol{\Omega}^{\mathrm{P}}=\sum_{\alpha=1}^{12} \frac{1}{2}\left(\boldsymbol{s}^{(\alpha)} \cdot \boldsymbol{m}^{(\alpha)}-\boldsymbol{m}^{(\alpha)} \cdot \boldsymbol{s}^{(\alpha)}\right) \dot{\gamma}^{(\alpha)}$,

where $\boldsymbol{s}^{(\alpha)}$ and $\boldsymbol{m}^{(\alpha)}$ are the slip direction and slip plane normal, respectively. In contrast, the crystal orientation changes during lattice $\operatorname{spin}\left(\boldsymbol{\Omega}^{*}\right)$. The plastic spin $\left(\boldsymbol{\Omega}_{\mathrm{TD}}^{\mathrm{P}}\right)$, lattice spin $\left(\boldsymbol{\Omega}_{\mathrm{TD}}^{*}\right)$, and material spin $\left(\boldsymbol{\Omega}_{\mathrm{TD}}\right)$ about the TD axis in matrix bands of roCube, Goss, and Copper are listed in Table 3.

In the roCube, the slip system set a1-a2 produced plastic spin about TD $\left(\boldsymbol{\Omega}_{\mathrm{TD}}^{\mathrm{P}}\right)$ in the positive direction according to Eq. (5), while the d1-d2 set caused negative $\boldsymbol{\Omega}_{\mathrm{TD}}^{\mathrm{P}}$. The $\boldsymbol{\Omega}_{\mathrm{TD}}^{\mathrm{P}}$ induced by $\gamma_{\mathrm{a} 1-\mathrm{a} 2}$ was partially or completely compensated by $\gamma_{\mathrm{b} 1-\mathrm{b} 2}$. The compensation is complete when $\gamma_{\mathrm{a} 1-\mathrm{a} 2}$ is equal to $\gamma_{\mathrm{b} 1-\mathrm{b} 2}$, and this can be found at the boundaries of adjacent matrix bands (Fig. 4 b), i.e. imbalance ratio being zero. Imbalance between $\gamma_{\mathrm{a} 1-\mathrm{a} 2}$ and $\gamma_{\mathrm{b} 1-\mathrm{b} 2}$ exists in matrix bands (Fig. 4 and Table 3), in which the compensation is partial. Due to the mutual compensation between slip system sets, the plastic spin is usually small when multi-slip occurs [12]. In M1, $\gamma_{\mathrm{b} 1-\mathrm{b} 2}$ is much higher than $\gamma_{\mathrm{a} 1-\mathrm{a} 2}$, and the compensation resulted in large negative $\boldsymbol{\Omega}_{\mathrm{TD}}^{\mathrm{P}}$. The total material spin $\left(\boldsymbol{\Omega}_{\mathrm{TD}}\right)$ represented by the shear strain $\left(\gamma_{X Y}\right)$ is negatively low (Fig. 3b). Therefore, positive lattice spin $\left(\boldsymbol{\Omega}_{\mathrm{TD}}^{*}\right)$ was required to meet the deformation according to Eq. (4). Though large $\boldsymbol{\Omega}_{\mathrm{TD}}^{*}$ evolved in the roCube, it is still lower than $\boldsymbol{\Omega}_{\mathrm{TD}}^{\mathrm{P}}$ (Table 3). Therefore, the shear strain is in the opposite direction to TD-rotation. This is how the redundant shear strain and TD-rotation are correlated. In M2, positive $\boldsymbol{\Omega}_{\mathrm{TD}}^{\mathrm{P}}$ was produced by $\gamma_{\mathrm{a} 1-\mathrm{a} 2}$ after being partially compensated by $\gamma_{\mathrm{b} 1-\mathrm{b} 2}$, and negative $\boldsymbol{\Omega}_{\mathrm{TD}}^{*}$ developed to meet the material spin $\boldsymbol{\Omega}_{\mathrm{TD}}$ (Table 3). The similar deformation behaviour was also observed in the Goss. The slip system set a2-a3 and c2-c3 produced positive and negative $\boldsymbol{\Omega}_{\mathrm{TD}}^{\mathrm{P}}$, respectively. In M1, $\gamma_{\mathrm{a} 2-\mathrm{a} 3}$ is higher than $\gamma_{\mathrm{c} 2-\mathrm{c} 3}$, so negative $\boldsymbol{\Omega}_{\mathrm{TD}}^{\mathrm{P}}$ was produced after their opposite contributions.

Table 3 Deformation in matrix bands of roCube, Goss, and Copper

\begin{tabular}{llllllll}
\hline Orientation & Matrix band & $\begin{array}{l}\text { Shear strain on slip } \\
\text { systems }\end{array}$ & $\boldsymbol{\Omega}_{\mathrm{TD}}^{\mathrm{P}}$ & $\boldsymbol{\Omega}_{\mathrm{TD}}^{*}$ & $\begin{array}{l}\text { Macroscopic shear } \\
\text { strain } \gamma_{X Y}\end{array}$ & $\boldsymbol{\Omega}_{\mathrm{TD}}$ & Relation $\boldsymbol{\Omega}_{\mathrm{TD}}=\boldsymbol{\Omega}_{\mathrm{TD}}^{\mathrm{P}}+\boldsymbol{\Omega}_{\mathrm{TD}}^{*}$ \\
\hline roCube & $\mathrm{M} 1$ & $\gamma_{\mathrm{a} 1-\mathrm{a} 2}<\gamma_{\mathrm{b} 1-\mathrm{b} 2}$ & $-\mathrm{TD}$ & $+\mathrm{TD}$ & - & $-\mathrm{TD}$ & $(-)=(-)+(+), \boldsymbol{\Omega}_{\mathrm{TD}}^{\mathrm{P}}>\boldsymbol{\Omega}_{\mathrm{TD}}^{*}$ \\
& $\mathrm{M} 2$ & $\gamma_{\mathrm{a} 1-\mathrm{a} 2}>\gamma_{\mathrm{b} 1-\mathrm{b} 2}$ & $+\mathrm{TD}$ & $-\mathrm{TD}$ & + & $+\mathrm{TD}$ & $(+)=(+)+(-), \boldsymbol{\Omega}_{\mathrm{TD}}^{\mathrm{P}}>\boldsymbol{\Omega}_{\mathrm{TD}}^{*}$ \\
Goss & $\mathrm{M} 1$ & $\gamma_{\mathrm{c} 2-\mathrm{c} 3}<\gamma_{\mathrm{a} 2-\mathrm{a} 3}$ & $-\mathrm{TD}$ & $+\mathrm{TD}$ & - & $-\mathrm{TD}$ & $(-)=(-)+(+), \boldsymbol{\Omega}_{\mathrm{TD}}^{\mathrm{P}}>\boldsymbol{\Omega}_{\mathrm{TD}}^{*}$ \\
& $\mathrm{M} 2$ & $\gamma_{\mathrm{c} 2-\mathrm{c} 3}>\gamma_{\mathrm{a} 2-\mathrm{a} 3}$ & $+\mathrm{TD}$ & $-\mathrm{TD}$ & + & $+\mathrm{TD}$ & $(+)=(+)+(-), \boldsymbol{\Omega}_{\mathrm{TD}}^{\mathrm{P}}>\boldsymbol{\Omega}_{\mathrm{TD}}^{*}$ \\
Copper & $\mathrm{M} 2$ & $\gamma_{\mathrm{a} 1-\mathrm{a} 2}>\gamma_{\mathrm{c} 3-\mathrm{d} 3}$ & $+\mathrm{TD}$ & $-\mathrm{TD}$ & + & $+\mathrm{TD}$ & $(+)=(+)+(-), \boldsymbol{\Omega}_{\mathrm{TD}}^{\mathrm{P}}>\boldsymbol{\Omega}_{\mathrm{TD}}^{*}$ \\
& $\mathrm{M} 1 \mathrm{a}$ & $\gamma_{\mathrm{a} 1-\mathrm{a} 2}>\gamma_{\mathrm{c} 3-\mathrm{d} 3}$ & $+\mathrm{TD}$ & $+\mathrm{TD}$ & + & $+\mathrm{TD}$ & $(+)=(+)+(+)$ \\
& $\mathrm{M} 1 \mathrm{~b}$ & $\gamma_{\mathrm{a} 1-\mathrm{a} 2}<\gamma_{\mathrm{c} 3-\mathrm{d} 3}$ & $-\mathrm{TD}$ & $+\mathrm{TD}$ & + & $+\mathrm{TD}$ & $(+)=(-)+(+), \boldsymbol{\Omega}_{\mathrm{TD}}^{\mathrm{P}}<\boldsymbol{\Omega}_{\mathrm{TD}}^{*}$ \\
\hline
\end{tabular}


According to Eq. (4), positive $\boldsymbol{\Omega}_{\mathrm{TD}}^{*}$ was required to meet the negative $\boldsymbol{\Omega}_{\mathrm{TD}}$. In contrast, $\boldsymbol{\Omega}_{\mathrm{TD}}^{\mathrm{P}}, \boldsymbol{\Omega}_{\mathrm{TD}}^{*}$, and $\boldsymbol{\Omega}_{\mathrm{TD}}$ in $\mathrm{M} 2$ are positive, negative, and positive, respectively. Similar to that in the roCube, the redundant shear strain is in the opposite direction to TD-rotation. In case $\mathrm{C}$, the activation of a1-a2 caused positive $\boldsymbol{\Omega}_{\mathrm{TD}}^{\mathrm{P}}$, and c3-d3 resulted in negative $\boldsymbol{\Omega}_{\mathrm{TD}}^{\mathrm{P}}$. In M2, the redundant $\boldsymbol{\Omega}_{\mathrm{TD}}^{\mathrm{P}}$ after the compensation is positively large due to the large difference between $\gamma_{\mathrm{a} 1-\mathrm{a} 2}$ and $\gamma_{\mathrm{c} 3-\mathrm{d} 3}$, and negative $\boldsymbol{\Omega}_{\mathrm{TD}}^{*}$ developed to accommodate $\boldsymbol{\Omega}_{\mathrm{TD}}$. In this matrix band, $\boldsymbol{\Omega}_{\mathrm{TD}}^{\mathrm{P}}$ is larger than $\boldsymbol{\Omega}_{\mathrm{TD}}^{*}$ (Table 3), so TD-rotation and shear strain are in the opposite direction. $\boldsymbol{\Omega}_{\mathrm{TD}}^{\mathrm{P}}$ became smaller in M1a due to the decreased difference between $\gamma_{\mathrm{a} 1-\mathrm{a} 2}$ and $\gamma_{\mathrm{c} 3-\mathrm{d} 3}$, and low positive $\boldsymbol{\Omega}_{\mathrm{TD}}^{*}$ evolved to meet the positive $\boldsymbol{\Omega}_{\mathrm{TD}}$. In M1b, negative $\boldsymbol{\Omega}_{\mathrm{TD}}^{\mathrm{P}}$ developed, since $\gamma_{\mathrm{a} 1-\mathrm{a} 2}$ is lower than $\gamma_{\mathrm{c} 3-\mathrm{d} 3}$. Large positive $\boldsymbol{\Omega}_{\mathrm{TD}}^{*}$ developed to accommodate the positive $\boldsymbol{\Omega}_{\mathrm{TD}} \cdot \boldsymbol{\Omega}_{\mathrm{TD}}^{*}$ is higher than $\boldsymbol{\Omega}_{\mathrm{TD}}^{\mathrm{P}}$ in M1b (Table 3), so shear strain and TD-rotation are in the same direction. In the Brass, the same activated slip systems having the same magnitude of shear strain is the reason for the uniform deformation in the whole sample. The large change in the sample geometry is caused by the crystal slip, which is not reduced by the lattice spin.

The correlation between crystal rotation and shear strain has not been extensively studied. A plasticity analysis was performed to study the correlation in Cube and Goss [3], in which a shear strain imbalance between the two sets of slip systems was introduced to generate the ' +-+- ' pattern, but the value of imbalance shear strain has not been validated. In contrast, the imbalance shear strain between slip system sets was accurately calculated in this study, and the imbalance ratio varies along the thickness (e.g. in Figs. 4b, 6b). The alternate slip system activation along the thickness is due to the influence of shear stress, since the shear stress would cause an imbalance of resolved shear stress between the two sets of slip systems. The shear stress is due to the combined effect of rolling bite geometry and surface friction $[33,34]$. The slip systems in the roCube are more sensitive to the shear stress due to their large inclination, which means a large difference in the resolved shear stress on slip systems between $\mathrm{a} 1-\mathrm{a} 2$ and $\mathrm{b} 1-\mathrm{b} 2$ is expected, and consequently, the imbalance ratio in Fig. 4b varies greatly. In contrast, the inclination of the activated slip systems in the Goss is comparatively low (Fig. 2b), and thus, the influence of shear stress to the resolved shear stress on slip systems is limited [30], so the shear strain on a2-a3 is close to $\gamma_{\mathrm{c} 2-\mathrm{c} 3}$. Besides, the plastic spin is also influenced by the slip system direction according to Eq. (5), where the change in slip system direction due to crystal rotation is not considered in the plasticity analysis in Ref. [3]. The shear strain on slip systems increases almost linearly with deformation time (Fig. 9) due to no crystal rotation. The plastic spin in the Goss would be higher than that in the roCube if the activated slip systems in them are of the same shear strain. Similarly, the plastic spin

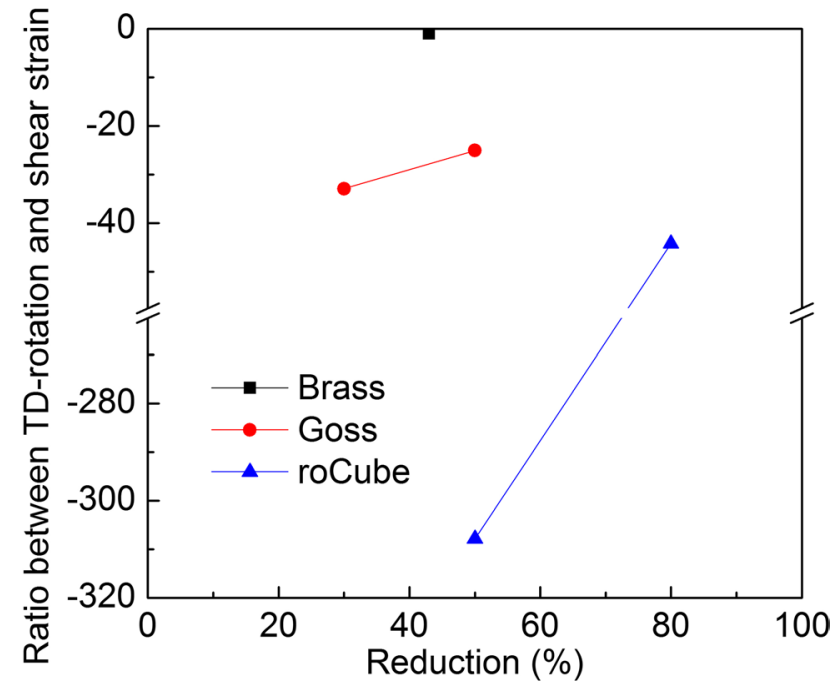

Fig. 10 Ratios between crystal rotation and redundant shear strain

would be even less in the Copper, and this is why the plastic spin is lower than lattice spin in M1b. Figure 10 shows the ratio between increase in TD-rotation and shear strain as a function of reduction in the Brass, Goss, and roCube. The ratio in the Brass is almost zero due to no crystal rotation, while it changes slowly in the Goss. The study in Ref. [6] shows that the crystal orientation does not change after it reached stability, and in this case, the plastic spin, or slip system activation, is the only deformation mechanism. The ratio in Fig. 10 decreases quickly with increasing strain in the roCube, which means the orientation rotates towards its stable position, and the role that plastic spin plays becomes dominant.

\section{Conclusions}

1. CPFE simulations of four single crystals have been conducted, and the predictions match well with the experimental observations.

2. Through-thickness deformation is inhomogeneous in the roCube, Goss, and Copper, and the different slip system activation, crystal rotation, and shear strain divide the thickness into matrix bands.

3. The shear strain distributes in the same manner as TDrotation in the roCube and Goss, but in opposite direction, since the plastic spin is higher than crystal rotation. Crystal rotation being higher than plastic spin has been found in M1b of the Copper, and accordingly, TD-rotation and shear strain are in the same direction.

4. Both crystal slip and crystal rotation develop in unstable orientations, and they compensate the material spin caused by each other, which thus lowers the redundant 
shear strain. The role of crystal slip becomes dominant when the orientations rotate towards stable positions.

Acknowledgements The simulation was performed on the HPC cluster of the University of Wollongong.

\section{References}

[1] H. Paul, J.H. Driver, Z. Jasieński, Acta Mater. 50, 815 (2002)

[2] N. Afrin, M.Z. Quadir, W. Xu, M. Ferry, Acta Mater. 60, 6288 (2012)

[3] Q. Liu, J. Wert, N. Hansen, Acta Mater. 48, 4267 (2000)

[4] H. Paul, J. Driver, C. Maurice, M. Miszczyk, D. Piot, Arch. Metall. Mater. 54, 65 (2009)

[5] K. Kashihara, H. Inagaki, Ceram. Trans. 201, 453 (2008)

[6] Z.J. Li, A. Godfrey, Q. Liu, Acta Mater. 52, 149 (2004)

[7] M. Wrobel, S. Dymek, M. Blicharski, S. Gorczyca, Mater. Res. Adv. Technol. 85, 415 (1994)

[8] Q. Liu, N. Hansen, Proc. R. Soc. A 454, 2555 (1998)

[9] J.A. Wert, Acta Mater. 50, 3125 (2002)

[10] P. Van Houtte, S. Li, M. Seefeldt, L. Delannay, Int. J. Plast. 21, 589 (2005)

[11] H.R. Wenk, P. Van Houtte, Rep. Prog. Phys. 67, 1367 (2004)

[12] C. Lu, G.Y. Deng, A.K. Tieu, L.H. Su, H.T. Zhu, X.H. Liu, Acta Mater. 59, 3581 (2011)

[13] C. Zhang, L.W. Zhang, W.F. Shen, Y.N. Xia, Y.T. Yan, Acta Metall. Sin. (Engl. Lett.) 30, 79 (2017)
[14] F. Roters, P. Eisenlohr, L. Hantcherli, D.D. Tjahjanto, T.R. Bieler, D. Raabe, Acta Mater. 58, 1152 (2010)

[15] D. Raabe, F. Roters, F. Barlat, L.-Q. Chen (eds.), Continuum Scale Simulation of Engineering Materials: Fundamentals-Microstructures-Process Applications (Wiley, Weinheim, 2004)

[16] K. Kashihara, Y. Tsujimoto, D. Terada, N. Tsuji, Mater. Charact. 75, 129 (2012)

[17] T. Inoue, N. Tsuji, Comput. Mater. Sci. 46, 261 (2009)

[18] A. Albou, J.H. Driver, C. Maurice, Acta Mater. 58, 3022 (2010)

[19] R.J. Asaro, J. Appl. Mech. 50, 921 (1983)

[20] R.J. Asaro, A. Needleman, Acta Metall. 33, 923 (1985)

[21] T.-Y. Wu, J.L. Bassani, C. Laird, Proc. R. Soc. A 435, 20 (1991)

[22] J.L. Bassani, T.-Y. Wu, Proc. R. Soc. A 435, 21 (1991)

[23] G. Lin, K.S. Havner, Int. J. Plast. 12, 695 (1996)

[24] P. Franciosi, M. Berveiller, A. Zaoui, Acta Metall. 28, 273 (1980)

[25] Q. Liu, C. Maurice, J. Driver, N. Hansen, Metall. Mater. Trans. A 29, 2333 (1998)

[26] D. Guanyu, Dissertation, University of Wollongong, 2014

[27] L.Y. Si, C. Lu, N.N. Huynh, A.K. Tieu, X.H. Liu, J. Mater. Process. Technol. 201, 79 (2008)

[28] P. Wei, C. Lu, H. Liu, L. Su, G. Deng, K. Tieu, Crystals 7, 12 (2017)

[29] A. Godfrey, D.J. Jensen, N. Hansen, Acta Mater. 46, 835 (1998)

[30] R.Y. Liang, P. Yang, W.M. Mao, Acta Metall. Sin. (Engl. Lett.) 30, 895 (2017)

[31] Z.S. Zhu, N.P. Chen, J.L. Gu, M.G. Yan, Acta Metall. Sin. Ser. A 9, 601 (1996)

[32] S. Li, F. Sun, H. Li, Acta Mater. 58, 1317 (2010)

[33] C.S. Lee, B.J. Duggan, Metall. Trans. A 22, 2637 (1991)

[34] J.X. Li, X.H. Liu, G.D. Wang, Acta Metall. Sin. (Engl. Lett.) 15, $312(2002)$ 